\title{
Calidad ósea en poblaciones contemporáneas. Análisis de la estructura trabecular calcánea en una muestra esqueletal
}

\section{Bone Quality in Contem porary Populations. Calcaneal Trabecular Structure Analysis in a Skeletal Sample}

\author{
Marcos Plischuk ${ }^{1,2}$ Gonzalo Garizoain ${ }^{1,2}$ Susana Salceda 2,3 \\ ${ }^{1}$ Facultad de Ciencias Médicas, Universidad Nacional de La Plata, \\ La Plata, Buenos Aires, Argentina \\ ${ }^{2}$ CONICET, Argentina \\ ${ }^{3}$ División Antropología, Facultad de Ciencias Naturales y Museo, \\ Universidad Nacional de La Plata, La Plata, Buenos Aires, Argentina

\begin{abstract}
Address for correspondence Marcos Plischuk, PhD, Cátedra de Citología, Histología y Embriología “A," Facultad de Ciencias Médicas, Universidad Nacional de La Plata, La Plata, Buenos Aires, Argentina (e-mail: marcosplischuk@yahoo.com.ar).
\end{abstract}

\section{Resumen}

\section{Palabras Clave}

- osteoporosis

- estructura trabecular

- calcáneo
Objetivo La osteoporosis es una condición de relevancia a nivel epidemiológico en Argentina, así como en el resto del mundo. Si bien actualmente la herramienta diagnóstica más relevante es la densitometría ósea, los análisis radiológicos pueden aportar información, principalmente en lo referente a la calidad ósea. En ese sentido, nos proponemos evaluar la estructura trabecular calcánea de una población local.

Métodos El material para dicho estudio consistió en 91 calcáneos de esqueletos pertenecientes a una muestra esqueletal. Fueron analizados individuos adultos, de ambos sexos, mediante imágenes radiográficas, a fin de clasificar su estructura trabecular de acuerdo al índice propuesto por Jhamaria y col., en una escala de $\mathrm{V}$ (normal) a I (osteoporosis severa).

Resultados Las prevalencias estimadas fueron de $23,1 \%$ de osteoporosis (fases I y II), $16,5 \%$ de borderline (fase III) y $48,4 \%$ se clasificó como estructura trabecular normal (fases IV y V). En relación a la edad de los individuos, se encontró una correlación significativa negativa entre ella y la pérdida trabecular (tau-b $=-0,524, p=0,00$ ). No se encontraron diferencias significativas en cuanto a la afección dimórfica de la patología. Discusión La relación entre envejecimiento y osteoporosis hallada es coincidente con estudios nacionales e internacionales que reconocen una disminución en la densidad mineral ósea (DMO) y una mala calidad ósea en individuos adultos mayores. La ausencia de afección dimórfica podría deberse a la edad promedio de la muestra, sumada a la posible resistencia de la estructura esponjosa del calcáneo a los cambios generados por el déficit estrogénico en las mujeres, característica de la osteoporosis tipo I.

Conclusión Creemos que es necesario revalorizar el análisis óseo por medio de radiografías, puesto que pueden ser de utilidad tanto en la clínica como en la investigación básica, debido a la mayor disponibilidad de equipos y a que posibilitan el análisis de otras propiedades del tejido óseo. received January 10,2020 accepted January 10, 2020
DOI https://doi.org/ 10.1055/s-0040-1708463. ISSN 1852-9992.
Copyright @ $\odot$ 2020, Sociedad Argentina de Radiología. Publicado por Thieme Revinter Publicações Ltda., Rio de Janeiro, Brazil. Todos los derechos reservados.
License terms

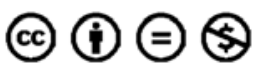




\section{Abstract}

\section{Keywords}

- osteoporosis

- trabecular meshwork

- calcaneus
Objective Osteoporosis is an important condition in an epidemiologic level in Argentina as in the rest of the world. Even though nowadays the most relevant diagnostic technique is bone densitometry, radiological studies can provide useful information, mainly in relation to bone quality. For this purpose, we aim to evaluate the calcaneus trabecular structure in a contemporary local population.

Methods The sample for this study consisted of 91 calcaneus from skeletons belonging to the skeletal repository. Adult individuals from both sexes were analyzed, to classify their trabecular structure according to the index proposed by Jhamaria et al., on a scale of $\mathrm{V}$ (normal) to I (severe osteoporosis).

Results The estimated prevalence were $23,1 \%$ of osteoporosis (phase I and II), $16,5 \%$ of borderline (phase III) and $48,4 \%$ were classified as normal trabecular structure (phase IV and V). In relation to the age of the individuals, a significant negative correlation was found between the first and the trabecular loss (tau-b $=-0.524, p=0.00$ ). No significant differences were found in relation to the sex prevalence of this pathology.

Discussion The association found between aging and osteoporosis matches with the results of national and international studies that recognize that a decrease in Bone Mineral Density (BMD) and a bad bone quality in older adults. Absence of differences between sexes in this condition could be due to age average of the sample, added to the possible resistance of the calcaneus spongy structure to changes generated by estrogen deficit in women, characteristic of osteoporosis type I.

Conclusion We believe that it is necessary to revalue bone analysis by radiographic means, since they can be of use both in clinic and in basic research, due to a greater equipment availability and because they allow the analysis of other properties of bone tissue.

\section{Introducción}

En 1994, un grupo de investigación de la Organización Mundial de la Salud (OMS), definió la osteoporosis como "una enfermedad esqueletal sistémica caracterizada por un descenso en la masa ósea y un deterioro en la microarquitectura del tejido óseo, con el consecuente incremento en fragilidad ósea y susceptibilidad a las fracturas." tancia de esa condición radica en que su presencia aumenta el riesgo de fracturas por fragilidad. ${ }^{2}$ Para dimensionar la problemática de esa patología en la salud pública en nuestro país, vale mencionar el estudio epidemiológico de Spivacow y Sanchez, ${ }^{3}$ que señala que un $50 \%$ de las mujeres mayores de 50 años presenta osteopenia y un $25 \%$ osteoporosis, se reportan más de 34.000 fracturas de cadera anuales y se estima una prevalencia del 16,2\% de fracturas vertebrales en ese grupo, sumado a la escasez de equipos densitométricos diagnósticos.

El descenso de los niveles de densidad mineral ósea (DMO) comienza hacia la tercera década de vida, y se estima en aproximadamente $0,5 \%$ por año. ${ }^{4,5}$ El pico de masa ósea alcanzado hacia los 30 años está determinado entre un 50 y un $80 \%$ por factores genéticos, a la vez que es influido por factores poblacionales, sexo, contextura, actividad física y hábitos nutricionales, entre otros. ${ }^{1,4-6}$ Sin embargo, tanto el nivel alcanzado como, principalmente, la tasa de resorción ósea posterior, se encuentran influidos por una multiplicidad de factores. Entre los más destacables mencionaremos el envejecimiento, amenorrea, menopausia, actividad física, his- torial familiar de osteoporosis, déficit nutricional (calcio, vitamina D), tabaquismo, alcoholismo y medicaciones (corticoides, anticonvulsionantes, anticoagulantes, antiácidos). ${ }^{5-12}$

En la actualidad, el método clínico más utilizado para su diagnosis es la absorciometría dual de rayos X (DEXA), que consiste en la evaluación de tejido mineralizado en un área escaneada $\left(\mathrm{g} / \mathrm{cm}^{2}\right)$ en uno o varios huesos por medio de una fuente de dos energías de rayos $\mathrm{X}$ que son absorbidos por los tejidos. ${ }^{13}$ Luego, esa absorción es analizada por un sistema informático, y la DMO es estimada por comparación con estándares de densidad conocida de mujeres blancas, sanas y jóvenes, considerando un T-score menor a -2,5 DS indicador de osteoporosis, mientras que entre -1 y $-2,5$ de osteopenia. 6,14 Sin embargo, la fortaleza ósea no se encuentra determinada solo por la densidad $(\mathrm{g} / \mathrm{cm} 2, \mathrm{~g} / \mathrm{cm} 3)$, sino también por la calidad ósea. Ese componente, conformado a su vez por la arquitectura ósea, la tasa de recambio, acumulación de daño y efectividad de la mineralización es, en muchas ocasiones, infravalorado en diagnósticos de osteoporosis. ${ }^{14}$ Aspectos relacionados a la calidad ósea, tales como el tamaño, forma e integridad de las fibras colágenas, así como el espesor y conectividad trabecular, afectan la fortaleza general del hueso $y$, por ende, inciden en el riesgo de fractura del paciente.

En los estudios realizados en muestras esqueletales humanas, la pérdida de calidad y cantidad de tejido óseo habitualmente se evalúa por medio de la observación de fracturas osteoporóticas, análisis histomorfométricos y a través de imágenes radiológicas. ${ }^{5,15-20}$ Esas últimas proveen información valiosa al momento de evaluar la arquitectura 
trabecular, uno de los componentes de la calidad ósea de los individuos. En ese sentido, algunos estudios involucran al fémur, considerando su epífisis proximal como área característica en el deterioro del tejido óseo ya descrito. ${ }^{19,21,22} \mathrm{El}$ calcáneo, en tanto, es otro elemento utilizado para el análisis, puesto que allí también se suceden modificaciones estructurales de carácter progresivo, susceptibles de ser clasificados y correlacionados con probabilidad de fracturas. ${ }^{2}$ Incluso fue utilizado en análisis radiológicos clínicos previos al uso masivo de la DEXA, puesto que, a diferencia del fémur, presentaba un posicionamiento más sencillo, sumado a una mayor distancia de las gónadas, lo que redundaba en una buena opción para evaluar un hueso expuesto de manera directa al peso corporal. ${ }^{23}$

En este hueso, el desbalance entre la acción de osteoblastos y osteoclastos genera una mayor resorción de tejido óseo, eliminando y adelgazando las trabéculas calcáneas del hueso conforme un patrón regular. Esa pérdida ósea genera modificaciones observables de los grupos trabeculares de tensión y compresión, dispuestos originalmente en respuesta, principalmente al peso corporal que debe soportar el calcáneo. ${ }^{24,25}$

A partir de lo expuesto, se planteó como objetivo de este trabajo analizar la pérdida de calidad ósea en una población contemporánea urbana a partir de sus restos esqueletales, particularmente evaluando la arquitectura trabecular calcánea.

\section{Materiales y Métodos}

\section{Bioética}

El proyecto de investigación que involucra la muestra esqueletal se encuadra en la legislación municipal vigente y está aprobado por el Comité de Bioética de la Institución donde se realizó el trabajo.

\section{Características de la muestra}

Se analizaron 91 calcáneos de lateralidad derecha, todos correspondientes a individuos seleccionados al azar de una muestra esqueletal compuesta por 298 individuos adultos. Todos los individuos que componen la colección presentan documentación asociada asentada en la institución que cedió los restos.

La muestra observada estuvo integrada por 46 casos de sexo masculino y 47 de sexo femenino, todos adultos entre los 18 y los 92 años (promedio de edad: 59,3 años). Las fechas de fallecimiento para la totalidad de la muestra esqueletal comprendieron el período 1900-2003, mientras que para los individuos analizados en el presente trabajo, comprendieron el período 1932-2001, siendo su nacionalidad mayoritariamente argentina.

\section{Metodología}

La evaluación de las modificaciones de la estructura trabecular calcánea permite valorar la calidad ósea, evaluando su espesor, longitud y conectividad. ${ }^{26-29}$ En un corte sagital del hueso pueden observarse dos grupos diferenciados de trabéculas, organización que responde a fuerzas de compresión y de tensión respectivamente. El primero de esos grupos comprende a su vez dos conjuntos menores de trabéculas, el primero con origen en la superficie articular subastragalina, que discurre hacia la zona posterior e inferior del hueso, evidenciando una forma de abanico. El segundo grupo de compresión, de menores dimensiones, se origina en la cara anterior de la superficie subastragalina y se dirige hacia la zona anterior del calcáneo, en la articulación calcáneo-cuboidea. El grupo tensil se distribuye en un conjunto primario de trabéculas, el cual nace en la cara inferior del hueso y corre hacia la zona superior y posterior, y uno secundario que se dirige a la carilla articular calcáneo-cuboidea. Ese último conjunto presenta trabéculas más delgadas y con mayor espacio entre las mismas, y llega a intersectarse con el segundo grupo de trabéculas de compresión.

Se decidió la utilización del método propuesto por Jhamaria y col., ${ }^{26}$ consistente en la identificación de cinco estadios para las modificaciones de dicha morfología en relación a la pérdida de DMO (-Fig. 1). Las características de cada fase son:

Fase V (normal): Las trabéculas compresivas y tensiles se encuentran normalmente distribuidas (-Fig. 2).

Fase IV (normal): Se nota la ausencia del sector medio del grupo compresivo primario, observándose solo dos pilares, uno superior y otro inferior.

Fase III (borderline): Se observa una disminución del grupo primario tensil, el cual sólo se cruza con el pilar inferior del grupo compresivo primario (-Fig. $\mathbf{3}$ ).

Fase II (osteoporosis): El grupo tensil secundario desaparece y el primario se reduce aún más.

Fase I (osteoporosis severa): Desaparecen ambos grupos tensiles. Las trabéculas de compresión se adelgazan y reducen en número (-Fig. 4).

Las radiografías para revelar la morfología trabecular calcánea fueron obtenidas a $50 \mathrm{kV}, 5 \mathrm{mAmper}$ y a $100 \mathrm{~cm}$ de distancia foco-película, en un equipo digital de alta definición Pimax 500 de un hospital local. Las imágenes fueron codificadas con el número de orden del individuo en la colección y evaluadas por uno de los autores con asistencia de un médico especialista en el diagnóstico por imágenes, que fue también el responsable de la obtención de la imagen. A fin de evaluar la confiabilidad de las observaciones realizadas, se realizó una prueba de error intraobservador mediante el índice de Kappa como medida de concordancia. ${ }^{30,31}$

\section{Análisis de los datos}

Los datos se examinaron a través de varias pruebas estadísticas. Al tratarse de un estudio analítico/observacional transversal, se relacionaron las observaciones con diversas variables consideradas factores de riesgo para la osteoporosis. En primer lugar, se analizó la prevalencia de los casos de osteoporosis en función del sexo. Para tal fin, se utilizó el test de Chi-cuadrado, a fin de evaluar la no independencia entre sendas variables. Por otro lado, se analizó la relación entre edad y la fase asignada utilizando la prueba de correlación de Tau-b de Kendall, con el objeto de conocer si la calidad ósea (evaluada a partir de categorías), varía en función de los grupos de edad. La prueba de Tau-b de Kendall permite correlacionar variables que no adoptan valores de intervalos y de las cuales no es posible asumir que presenten distribución normal. ${ }^{32} \mathrm{Al}$ mismo tiempo, se calculó la categoría 

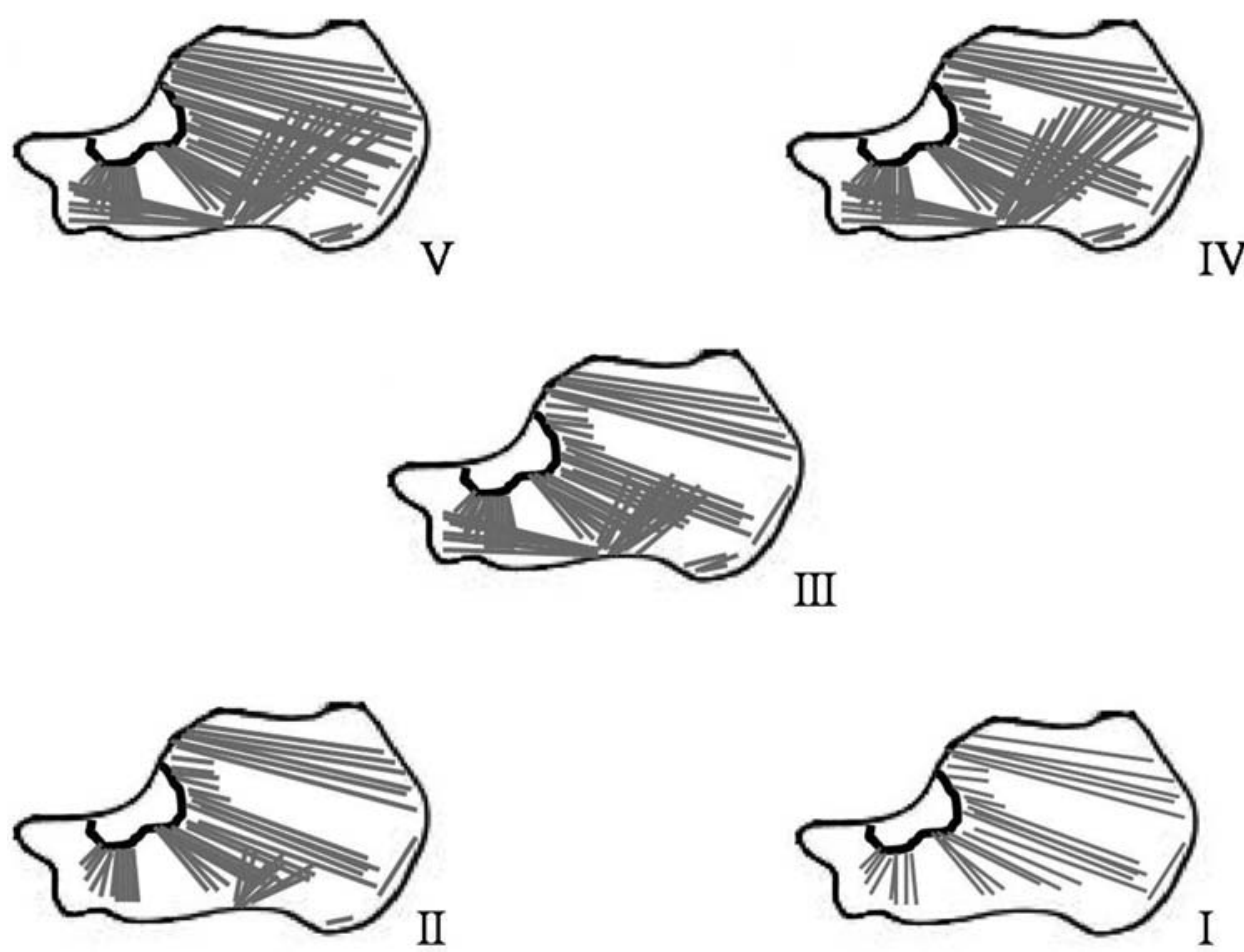

Fig. 1 Fases en las modificaciones de la estructura trabecular calcánea propuestas por Jhamaria y col. Fase I: osteoporosis severa. Fase II: osteoporosis. Fase III: borderline. Fases IV y V: normal.

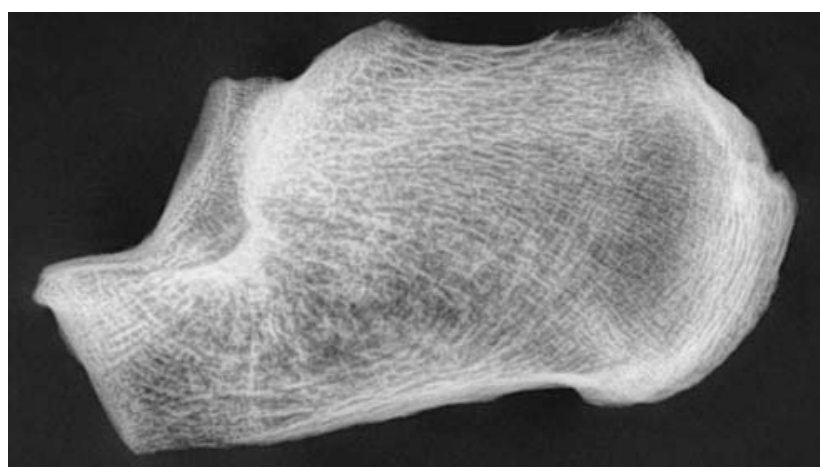

Fig. 2 Fase $V$ del índice trabecular calcáneo.

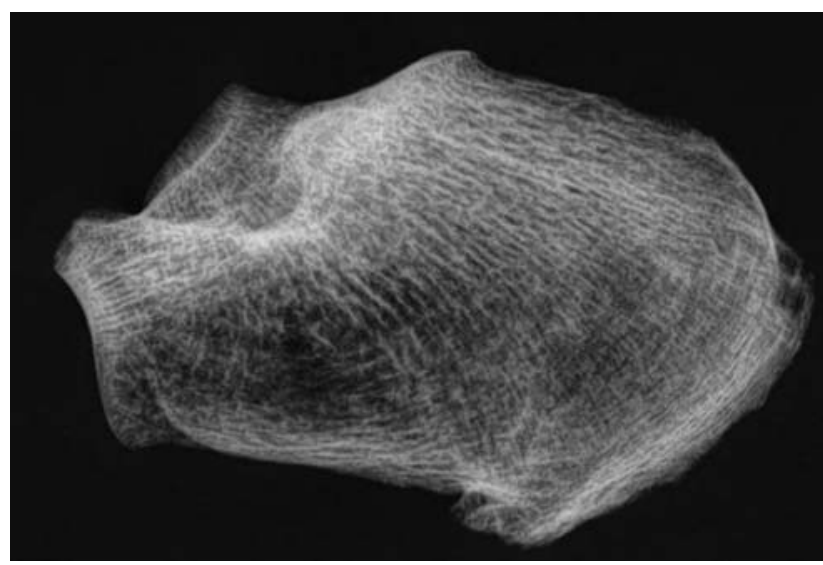

Fig. 3 Fase III del índice trabecular calcáneo.

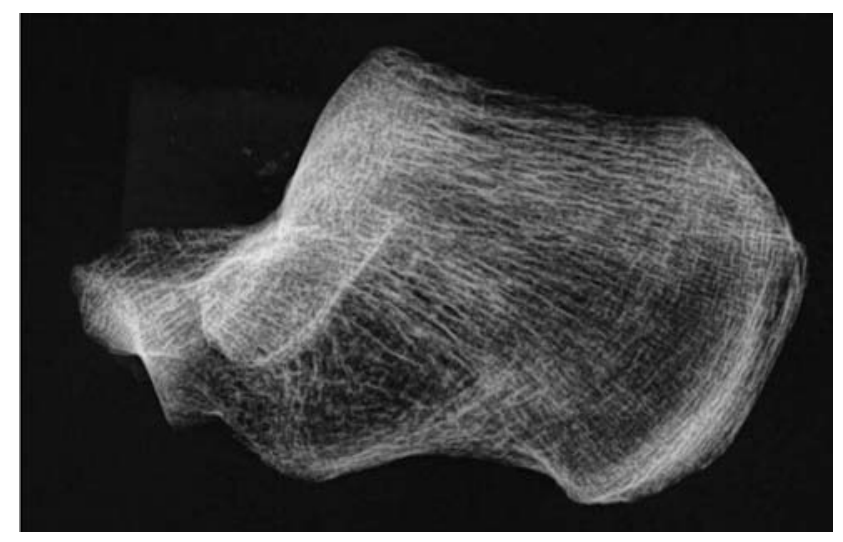

Fig. 4 Fase I del índice trabecular calcáneo.

promedio para los individuos agrupados en rangos de 10 años, y se comparó con lo obtenido por Jhamaria y col., ${ }^{26}$ en el trabajo original de 1983. Todos los análisis se realizaron con el programa de análisis estadístico IBM SPSS Statistics (SPSS Inc., released in 2008. SPSS Statistics for Windows, Version 17.0., Chicago) y el nivel de significación fue de 0,05.

\section{Resultados}

En primer lugar, el índice de Kappa obtenido tuvo un valor de 0,787 , lo que indicaría una buena concordancia entre set de observaciones. $^{30}$

Las prevalencias estimadas fueron de $23,1 \%$ de osteoporosis (fases I y II), siendo de 6,6\% para osteoporosis severa (fase I) y de 16,5\% para osteoporosis (fase II). La frecuencia de 
Tabla 1 Frecuencias por categoría del índice trabecular calcáneo

\begin{tabular}{|l|l|l|l|l|}
\hline \multicolumn{2}{|c|}{} & \multicolumn{2}{|l|}{ Sexo } & \multirow{2}{*}{ Total } \\
\cline { 3 - 5 } \multicolumn{2}{c|}{} & F & M & \\
\hline \multirow{4}{*}{ Fase } & I & 3 & 3 & 6 \\
\cline { 2 - 5 } & II & 8 & 7 & 15 \\
\cline { 2 - 5 } & III & 14 & 12 & 26 \\
\cline { 2 - 5 } & IV & 16 & 9 & 25 \\
\cline { 2 - 5 } & V & 5 & 14 & 19 \\
\hline Total & 46 & 45 & 91 \\
\hline
\end{tabular}

Referencias: F, femenino; M, masculino.

casos borderline (fase III) fue estimada en $28,6 \%$, mientras que el restante $48,4 \%$ se clasificó como estructura trabecular normal $(27,5 \%$ para la fase IV y $20,9 \%$ para la fase $V$ ) (-Tabla 1).

En relación a la edad de los individuos, aquellos clasificados como fase I (osteoporosis severa) presentaron una media de edad de 73,3 años y un desvío estándar (DS) de 7,20; en tanto que los agrupados en fase II (osteoporosis) mostraron una media de 76 años un DS de 8,97. Los casos clasificados como fase III (borderline) presentaron una media de 64,5 años y un DS de 13,15. Por último, aquellos que fueron clasificados fases IV y V (normales) presentaron una media de 49,8 +/-14,40 y 46,9+/-15,28 años de edad respectivamente. La correlación tau-b de Kendall para determinar la relación entre los estadios de Jhamaria y col., ${ }^{26}$ y la edad de los pacientes mostró una correlación negativa, que fue estadísticamente significativa ( $\tau b=$ $0,524, p=0,000$ ), lo cual indica que a medida que aumenta la edad los individuos sufren un adelgazamiento y pérdida de las trabéculas calcáneas. (-Fig. 5). En la - Tabla 2 se observa como a medida que los individuos envejecen la clasificación para su rango etario disminuye, significando una pérdida trabecular calcánea. Por otro lado, no se
Tabla 2 Relación entre el índice promedio para cada rango de edad entre este trabajo y la propuesta original de Jhamaria y col

\begin{tabular}{|l|l|l|l|}
\hline & \multicolumn{2}{|l|}{ Índice promedio } \\
\hline $\begin{array}{l}\text { Grupo de } \\
\text { edad }\end{array}$ & $\begin{array}{l}\text { Media de } \\
\text { edad }\end{array}$ & $\begin{array}{l}\text { Este } \\
\text { trabajo }\end{array}$ & $\begin{array}{l}\text { Jhamaria } \\
\text { col. }\end{array}$ \\
\hline-40 & 31,3 & 4,4 & 4,6 \\
\hline $41-50$ & 47,2 & 4,3 & 2,6 \\
\hline $51-60$ & 55,6 & 3,9 & 2,4 \\
\hline $61-70$ & 65 & 2,9 & 2,2 \\
\hline $71+$ & 79,6 & 2,4 & 1,7 \\
\hline
\end{tabular}

encontraron diferencias significativas en cuanto a la afección dimórfica de la patología. En ese sentido, el resultado del test de Chi-cuadrado no evidenció la existencia de una diferencia estadísticamente significativa en el porcentaje de osteoporosis en función del sexo $\left(X^{2}=10,12 \mathrm{gl}=4\right.$ $p=0,030)$. Al segmentar la muestra en individuos mayores y menores a los 60 años, tampoco se observan diferencias entre las fases asignadas a cada sexo, aunque es de destacar que no se observaron individuos de ningún sexo menores a esa edad límite en fase I o II.

\section{Discusión}

Históricamente, la evaluación del deterioro y fragilidad ósea a partir del análisis de material esqueletal se ha realizado a partir de diversas aproximaciones, puesto que la observación macroscópica puede ser problemática en tanto un hueso con apariencia externa normal pueda presentar osteopenia y pasar desapercibida. ${ }^{32}$ Es necesario, entonces, evaluar también el inicio de la pérdida de calidad ósea en casos de osteopenias leves, y los métodos para lograrlo necesariamente deben tener en cuenta la estructura trabecular del hueso analizado.

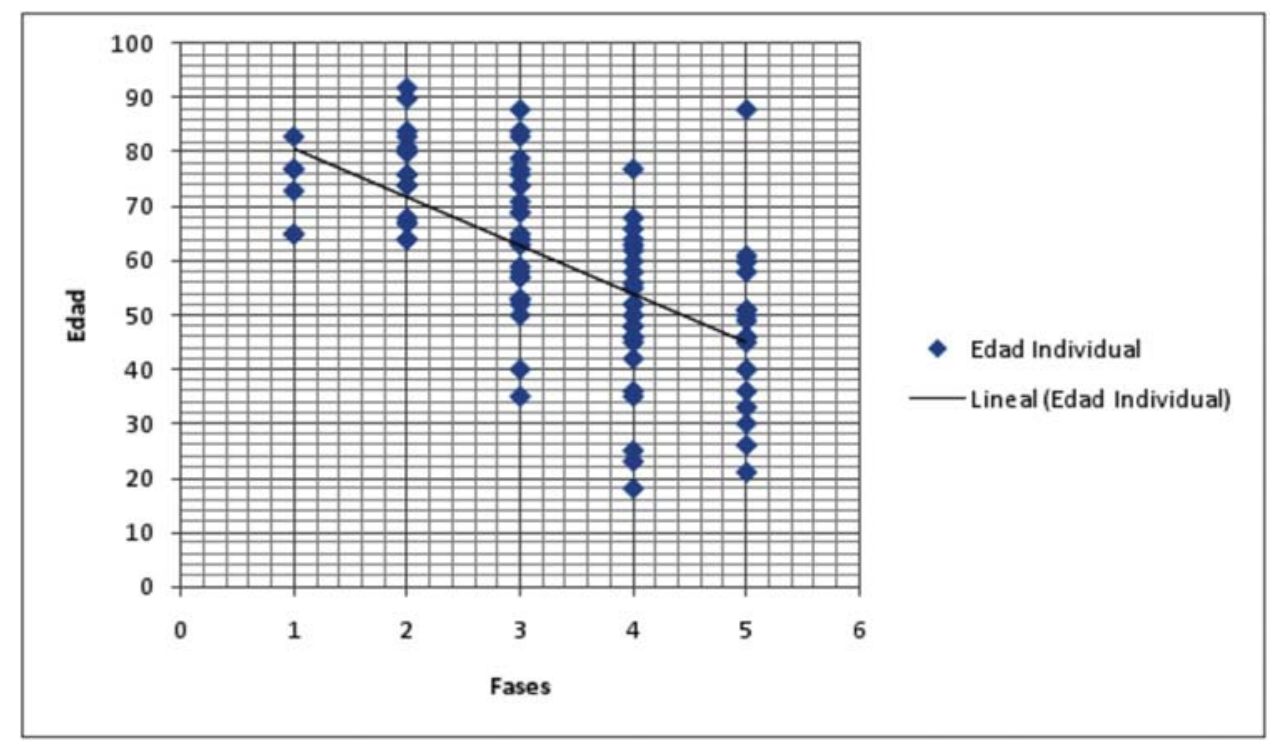

Fig. 5 Asociación entre las fases de pérdida de calidad ósea y la edad cronológica individual. 
En los resultados obtenidos se observa una correlación entre la pérdida de trabéculas calcáneas y la edad de los individuos. Esa tendencia es coincidente con múltiples estudios nacionales e internacionales que reconocen una disminución en la DMO y una mala calidad ósea en individuos adultos mayores. ${ }^{33-35}$ En el trabajo original de Jhamaria y col., ${ }^{26}$ ese hecho es observable en la clasificación promedio por grupo de edad, comprendiendo desde 4 en menores a 40 años a 1,75 en mayores de 71 ( - Tabla 2). Nuestros resultados muestran la misma tendencia, sin llegar a valores tan bajos en el grupo de mayor edad, eso último probablemente debido a los avances en el tratamiento de la osteoporosis, teniendo en cuenta que el trabajo de Jhamaria y col., ${ }^{26}$ fue realizado en 1983. Blake y col., ${ }^{35}$ en tanto, también encontraron una correlación entre la disminución de masa ósea y envejecimiento a partir de estudios densitométricos en calcáneo. Por último, Gupta y col., ${ }^{28}$ mediante la comparación del análisis radiológico trabecular de calcáneo con observaciones histológicas, concluyeron que la perdida trabecular descripta por Jhamaria y col., ${ }^{26}$ refleja de manera fiel el envejecimiento óseo. Si bien otros autores, como Pande y col., ${ }^{27}$ agruparon las fases I y II por un lado y IV y V por otro, alegando la dificultad de discernir entre dichas fases, en nuestro caso no se encontraron inconvenientes para identificarlas, y los resultados permitirán una comparación más sencilla con estudios previos.

El envejecimiento provoca un déficit en la estructura ósea tanto en su calidad como en su densidad, y ello se debe a una multiplicidad de factores asociados. En primer lugar, se observa una disminución en la eficiencia de las unidades básicas multicelulares (BMU) de remodelación ósea, en las cuales los depósitos de hueso por parte de los osteoblastos son menores al hueso reabsorbido por los osteoclastos siendo una de las causas una mayor tendencia adipogénica en la médula ósea. ${ }^{36-38}$

En segundo lugar, la disminución de ciertas hormonas influyentes en la fisiología ósea puede generar un hueso osteoporótico. Los estrógenos inhiben la resorción ósea, incidiendo en la diferenciación de precursores de osteoclastos, inhibiendo la producción de Receptor Activador para Factor Nuclear к B Ligando (RANK-L) por los osteoblastos e induciendo la producción de osteoprotegerina.

Por último, cabe destacar una serie de factores ambientales relacionados al envejecimiento que son potencialmente perjudiciales en la calidad y cantidad de tejido óseo en el esqueleto, característicos de una población urbana y contemporánea de edad avanzada. La actividad física es un agente que actúa estimulando la formación y orientación de las trabéculas óseas en función de los requerimientos mecánicos. ${ }^{10,12,32}$ Por su parte, hábitos como el tabaquismo, alcoholismo y consumo de fármacos actúan disminuyendo la reserva cálcica e impidiendo la normal absorción del calcio, aumentando la tasa de recambio y resorción ósea., ${ }^{7,8}$

En relación a la pérdida de tejido óseo durante la adultez, se esperaría encontrar que el descenso en los niveles de cantidad y calidad ósea sea más temprano en las mujeres, principalmente por la ya descrita acción estrogénica en el metabolismo óseo (osteoporosistipo I oposmenopáusica).
Sin embargo, al observar nuestro análisis no se encuentran diferencias en cuanto a la afección de los individuos femeninos de manera más temprana. Una explicación posible es que las trabéculas afectadas debido a la osteoporosis postmenopáusica son principalmente las vertebrales, ocasionando aplastamiento de los cuerpos. ${ }^{32}$ Así, la estructura trabecular calcánea no estaría evidenciando esa primera pérdida de tejido óseo en el sexo femenino. A edades más avanzadas, la osteoporosis senil afectaría a individuos de ambos sexos sin que se observe un aumento en el deterioro trabecular en relación directa con la edad. Eso explicaría que la media de edad del grupo II (osteoporosis) sea levemente mayor que la del grupo I (osteoporosis severa).

Por último, creemos que es importante discutir las dificultades y potencialidades del método y la muestra utilizada. Podemos sostener que las principales limitantes del método tienen que ver con la subjetividad del mismo, en tanto no se obtiene un valor numérico de DMO, y con la discutible representatividad del hueso elegido para estimar la salud ósea de un individuo o población. Sin embargo, el uso de la radiografía tradicional se ve revalorizado por análisis como el de Luo y col., ${ }^{39}$ en el que se observa una alta correlación $(r=0,99)$ entre las imágenes obtenidas y la estructura tridimensional del hueso. En el caso particular del método trabecular calcáneo, Gupta y col. ${ }^{28}$ testearon en pacientes vivos la especificidad (probabilidad de que un sujeto sano tenga un resultado negativo en la prueba), y sensibilidad (probabilidad de clasificar correctamente a un individuo enfermo), de diversos índices radiográficos para evaluar la osteoporosis. Los huesos evaluados fueron fémur, costillas, metacarpos y calcáneo, ese último de acuerdo a Jhamaria y col., ${ }^{26}$ método utilizado también en nuestro trabajo. Este método arrojó un $100 \%$ de especificidad y $58 \%$ de sensibilidad, convirtiéndolo en una opción a considerar al momento de evaluar pérdida de calidad ósea a través de imágenes radiográficas. A pesar de las aparentes limitaciones técnicas, el índice calcáneo pareciera ser capaz de diferenciar pacientes con baja DMO de aquellos con una DMO normal. Estudios densitométricos de esa índole fueron diseñados por nuestro equipo de trabajo y serán llevados a cabo en un futuro cercano. En cuanto a las limitaciones concernientes a la muestra utilizada, podemos establecer dos fuentes de error principales. Existe la posibilidad de que las modificaciones trabeculares observadas sean parcialmente ocasionadas por procesos diagenéticos, puesto que los restos óseos analizados habían estado enterrados durante largos períodos de tiempo. En ese sentido, recomendamos como criterio de inclusión seleccionar aquellos elementos con su cortical intacta, para minimizar el impacto de los agentes tafonómicos en el área esponjosa. En la actualidad, el equipo de trabajo se encuentra evaluando las características geomorfológicas del cementerio de origen para tener un mayor control sobre dichas variables. Por su parte, es claro que, al carecer de información acerca de la salud y hábitos del individuo, pueden generarse falsos positivos y/o negativos, aunque resaltamos que el objetivo de este análisis es el de observar tendencias poblacionales a partir de un material poco utilizado en ese sentido, como son las colecciones osteológicas. 


\section{Conclusión}

Por todo lo anteriormente expuesto, creemos que el análisis de las modificaciones de la estructura trabecular puede ser de utilidad tanto en la clínica como en la investigación básica, puesto que existe cada vez un mayor reconocimiento en las dificultades que tienen los estudios por DEXA como predictor único para el riesgo de fracturas, más allá de su indudable poder diagnóstico. ${ }^{1,14,29,35,39,40}$ El análisis de restos óseos humanos contemporáneos se suma así a investigaciones clínicas y experimentales sobre osteoporosis, contribuyendo a la generación de información al momento de analizar la salud ósea de una población.

\section{Responsabilidades Éticas}

Protección de personas y animales. Los autores declaran que para esta investigación no se han realizado experimentos en seres humanos ni en animales.

Confidencialidad de los datos. Los autores declaran que han seguido los protocolos de su centro de trabajo sobre la publicación de datos de pacientes.

Derecho a la privacidad y consentimiento informado. Los autores declaran que en este artículo no aparecen datos de pacientes.

\section{Reconocimientos}

Al Hospital Interzonal General de Agudos Prof. Dr. Rodolfo Rossi de la ciudad de La Plata, y en particular a Santiago Castilla y Jorgelina Falasco, por la asistencia en la toma de imágenes radiográficas. Al personal del Cementerio Municipal La Plata.

\section{Conflicto de Intereses}

Los autores declaran no tener ningún conflicto de intereses, excepto la coautoría de trabajos que se citan en el presente manuscrito.

\section{Bibliografía}

1 McCormick RK. Osteoporosis: integrating biomarkers and other diagnostic correlates into the management of bone fragility. Altern Med Rev. 2007;12(02):113-145

2 Roig Vilaseca D. Concepto y etiología de la osteoporosis. En: Arboleya Rodríguez L, Perez Edo L. eds. Manual de enfermedades óseas. Madrid: Editorial Médica Panamericana; 2010:171-176

3 Spivacow FR, Sánchez A. Epidemiology, costs, and burden of osteoporosis in Argentina, 2009. Arch Osteoporos. 2010;5(1-2): 1-6

4 Alonso-Bouzon C, Duque G. Osteoporosis senil: una actualización. Rev Esp Geriatr Gerontol. 2011;46(04):223-229

5 Brickley M, Agarwal S. Techniques for the investigation of agerelated bone loss and osteoporosis in archaeological bone. En: Agarwal SC, Stout SD, eds. Bone loss and osteoporosis: an anthropological perspective. Nueva York: Kluwer Plenum Academic Press; 2003:157-172

6 Schurman L, Bagur A, Claus-Hermberg H, Messina O, Negri A, Sánchez A, et al. Guías 2012 para el diagnóstico, la prevención y el tratamiento de la osteoporosis. Actual Osteol. 2013;9(02):123-153

7 Delezé MH, Morales J, Jara L. Osteoporosis inducida por corticosteroides patogenia, prevención y tratamiento. Rev Perú Reum. 1996;2(02):69-66
8 van Staa TP, Leufkens HG, Cooper C. The epidemiology of corticosteroid-induced osteoporosis: a meta-analysis. Osteoporos Int. 2002;13(10):777-787

9 Salica D. Osteoporosis inducida por tabaco. Rev Arg Osteol. 2003; 2:20-29

10 Krall EA, Dawson-Hughes B. Smoking increases bone loss and decreases intestinal calcium absorption. J Bone Miner Res. 1999; 14(02):215-220

11 Kanis JA, Johnell O, Oden A, Johansson H, De Laet C, Eisman JA, et al. Smoking and fracture risk: a meta-analysis. Osteoporos Int. 2005;16(02):155-162

12 Rodríguez Pérez M. Factores de riesgo de la osteoporosis y de las fracturas de naturaleza osteoporótica. En: Arboleya Rodríguez L, Perez Edo L eds. Manual de enfermedades óseas. Madrid: Editorial Médica Panamericana; 2010:177-184

13 Stride PJ, Patel N, Kingston D. The history of osteoporosis: why do Egyptian mummies have porotic bones? J R Coll Physicians Edinb. 2013;43(03):254-261

14 Martin RM, Correa PH. Bone quality and osteoporosis therapy. Arq Bras Endocrinol Metabol. 2010;54(02):186-199

15 Curate F. Osteoporosis and paleopathology: a review. J Anthropol Sci. 2014;92:119-146

16 Curate F, Albuquerque A, Correia J, Ferreira I, de Lima JP, Cunha EM. A glimpse from the past: osteoporosis and osteoporotic fractures in a Portuguese identified skeletal sample. Acta Reumatol Port. 2013;38(01):20-27

17 Curate F, Silva T, Cunha E. Vertebral compression fractures: Towards a standard scoring methodology in paleopathology. Int J Osteoarchaeol. 2014;26(02):362-372

18 Schultz M. Paleohistopathology of bone: a new approach to the study of ancient diseases. Am J Phys Anthropol. 2001;33(Suppl 33):106-147

19 Plischuk M, Inda A, Errecalde A. Modificaciones de la estructura ósea del fémur proximal. Análisis de una muestra esqueletal. Rev Argent Radiol. 2014;78(01):42-48

20 Mays S. Radiography and allied techniques in the palaeopathology of skeletal remains. En: Pinhasi R, Mays S eds Advances in human palaeopathology. Cichester: Wiley \& Sons; 2008:77-100

21 Singh M, Nagrath AR, Maini PS. Changes in trabecular pattern of the upper end of the femur as an index of osteoporosis. J Bone Joint Surg Am. 1970;52(03):457-467

22 Koot VC, Kesselaer SM, Clevers GJ, de Hooge P, Weits T, van der Werken C. Evaluation of the Singh index for measuring osteoporosis. J Bone Joint Surg Br. 1996;78(05):831-834

23 Banks A, Castellano N. Radiology of osteoporosis evaluation and interpretation. Proceedings of the Annual Meeting of the Podiatry Institute. Update. 1987;16:21

24 Ouellette $H$, Tetreault P. Clinical radiology made ridiculously simple. Miami: MedMaster, Inc.; 2008

25 Jhamaria NL, Lal KB, Udawat M, Banerji P, Kabra SG. The trabecular pattern of the calcaneum as an index of osteoporosis. J Bone Joint Surg Br. 1983;65(02):195-198

26 Malouf J, Laiz A, Farrerons J. Radiología de las enfermedades metabólicas óseas. En: Riancho Moral J, González Macías J, eds. Manual práctico de osteoporosis y enfermedades del metabolismo mineral. Madrid: Jarpyo Editores; 2004:77-82

27 Pande KC, Pande SK, de Takats D, McCloskey EV. Modified calcaneal index: a new screening tool for osteoporosis based on plain radiographs of the calcaneum. J Orthop Surg. 2005;13(01):27-33

28 Gupta G, Rani S, Umar S, Singh S, Haider S, Kumar M. Correlate of radiological indexes for osteoporosis with histological status of osteoid of bone. JDMS. 2016;15(04):20-28

29 Ortner D. Identification of pathological conditions in human skeletal remains. Nueva York: Academic Press; 2003

30 López de Ullibarri Galparsoro I, Pita Fernandez S. Medidas de Concordancia: el Indice de Kappa. Cad Aten Primaria. 1999; 6:169-171 
31 Romdhani H, Lakhal-Chaieb L, Rivest PL. Kendall's Tau for hierarchical data. J Multivariate Anal. 2014;128:210-225

32 Brickley M, Ives R. The bioarchaeology of metabolic bone disease. Oxford: Academic Press; 2008

33 Mensforth RP, Latimer BM. Hamann-Todd Collection aging studies: osteoporosis fracture syndrome. Am J Phys Anthropol. 1989; 80(04):461-479

34 Duque G, Troen BR. Understanding the mechanisms of senile osteoporosis: new facts for a major geriatric syndrome. J Am Geriatr Soc. 2008;56(05):935-941

35 Blake GM, Chin DJ, Steel SA, Patel R, Panayiotou E, Thorpe J, et al. National Osteoporosis Society Bone Densitometry Forum. A list of device-specific thresholds for the clinical interpretation of peripheral x-ray absorptiometry examinations. Osteoporos Int. 2005; 16(12):2149-2156

36 Raisz LG, Seeman E. Causes of age-related bone loss and bone fragility: an alternative view. J Bone Miner Res. 2001;16(11):1948-1952
37 Musacchio E, Priante G, Budakovic A, Baggio B. Effects of unsaturated free fatty acids on adhesion and on gene expression of extracellular matrix macromolecules in human osteoblast-like cell cultures. Connect Tissue Res. 2007;48(01): 34-38

38 Docheva D, Popov C, Mutschler W, Schieker M. Human mesenchymal stem cells in contact with their environment: surface characteristics and the integrin system. J Cell Mol Med. 2007;11 (01):21-38

39 Macchiarelli R, Bondioli L. Linear densitometry and digital image processing of proximal femur radiographs: implications for archaeological and forensic anthropology. Am J Phys Anthropol. 1994;93(01):109-122

40 Luo G, Kinney JH, Kaufman JJ, Haupt D, Chiabrera A, Siffert RS. Relationship between plain radiographic patterns and threedimensional trabecular architecture in the human calcaneus. Osteoporos Int. 1999;9(04):339-345 